\title{
A Study of the Subcortical Insect Community in Pine Trees. III. Species Correlation
}

\author{
Ken YoshiKawa \\ Faculty of Agriculture, Kochi University, Nankoku, Kochi 783, Japan
}

(Received October 20, 1986)

\begin{abstract}
Species correlations in the subcortical community of Pinus densiflora were analyzed with indices of $\gamma$ and $\omega$ proposed by Iwao (1977). All pine bait logs were attacked by Shirahoshizo spp. and Taenioglyptes fulvus because of their long active period. The basic distribution pattern in the trunk of Pinus densiflora was T. fulvus at upper part of the stem and Shirahoshizo spp. at the bottom. Although Pissodes nitidus attacked trees that lost their vigor in the spring and had the same distribution range with $T$. fulvus, the two species were distributed independently in each trunk unit and different niches were suggested by correlation. When pine trees weakened in the summer, two Cerambycidae participated in the community instead of $P$. nitidus. Monochamus alternatus and Acanthocinus griseus showed an overlapping distribution and competed severely with each other. However, $M$. alternatus usually won. $T$. fulvus seldom suffered from the presence of other species at the habitat available for development. The basic distribution pattern of the species was exclusion with each other in each trunk unit, but extreme difference in body size made possible coexistence with other species in the same habitat.
\end{abstract}

\section{INTRODUCTION}

There is a definite succession of organisms in logs as the chemical and physical character of the wood changes during the processes of disintegration and decay, beginning with xylophagous forms and concluding with an association of organisms characteristic of the duff strata of forest soils (GraHAM, 1925). In this study only the xylophagous stage was considered. Shirahoshizo spp. and Pissodes nitidus were the major species of Curculionidae emerging from bait logs. Emerging in smaller numbers were two Cerambycidae (Monochamus alternatus and Acanthocinus griseus) and one Scolytidae (Taenioglyptes fulvus). $M$. alternatus is the main vector of the pine wood nematode, Bursaphelenchus xylophilus, the pathogen involved in pine wilt disease (Kryohara and Tokushige, 1971; Kobayashi, 1978, 1981 ; Mamiya and Enda, 1972; Mamiya and Kiyohara, 1972).

Species menu of each community is governed by the relation between the period of insect attacks and the physiological degradation of the tree, usually beginning with the stop of resin secretion which makes the tree suitable for insect attack. From the bait logs prepared in early spring, a large number of $P$. nitidus emerged but few Cerambycidae ( $M$. alternatus and $A$. griseus) emerged. However, in early summer a large number of the two Cerambycidae emerged.

There are many studies on the ecology of bark beetles emphasizing interspecific correlations between host and parasite or prey and predator (BERRYMAN, 1966, 1967, 
1968, 1973; BeAver, 1966). However, among the rest, intra-guild competition, which is interspecific competition among species having same niche, is important for bark beetles community structure. After establishment of larval populations under bark, most are well protected from natural enemies. One of the main mortality factors for such insects is cannibalism or accidental predation by other xylophagous insects (YosHIKAWA, 1977). Most of the subcortical species have evolved ways to avoid intra-guild competition, for example character divergence.

In the first paper of this series, the biology of each species was reported and the vertical distributions were described in the second paper. In this paper, I analyze species correlations in the subcortical community by considering their life histories.

\section{METHODS}

Fourteen pine bait logs (mean D.B.H. was $11.1 \mathrm{~cm}$ and mean height was $8.5 \mathrm{~m}$ ) were prepared in a Japanese red pine forest at Takatori town in Nara Prefecture. All branches were taken off and the trunk divided into $50 \mathrm{~cm}$ long sections (trunk unit) was placed standing by roping to a live pine tree. The study area and materials were the same as those described in former papers of this series (Yoshikawa et al., 1986, YoshiKAWA, 1987). For the analysis of species correlations, I used only the results of treatments exposed to insect attacks from March 29 and June 20. Trunk units were attacked immediately after placement for various months from one to eight.

Many indices of "niche overlap" have been proposed to measure the degree of overlapping or spatial association between two species (DiGE, 1945; Cole, 1949; Morishita, 1959; Levins, 1968). In this paper, $\gamma$ and $\omega$ indices proposed by Iwao (1977) were calculated for each combination shown in Table 1 by the following equations;

$$
r=\sqrt{\frac{m_{x y} * *^{*}}{\left(m_{x x}+1\right)\left(\stackrel{*}{m}_{y}+1\right)}}
$$

where $\stackrel{*}{m}_{x}$ and $\stackrel{*}{m}_{y}$ are the mean crowding of species $x$ and species $y$ and $\stackrel{*}{m}_{x y}$ and $\stackrel{*}{m}_{y x}$ are the mean crowding on species $x$ by species $y$ and vice versa. The $\gamma$ index takes the maximum value of 1.0 when the distributions of both species are completely overlapped and the minimum value of 0.0 when the distributions are completely exclusive from each other. The $\gamma$ index expected for independent distributions of two species is

$$
r(\text { ind })=\sqrt{\frac{m_{x}}{\left(m_{x}+1\right)} * \frac{m_{y}}{\left(m_{y}^{*}+1\right)}}
$$

where $m_{x}$ and $m_{y}$ are the mean density of species $x$ and species $y$. Then, as a measure of the degree of spatial correlation relative to the independent distributions, we have when $r \geq r$ (ind)

$$
\omega(+)=\frac{r-r(\text { ind })}{1-r(\text { ind })}
$$

or when $r<\gamma($ ind $)$

$$
\omega(-)=\frac{r-r(\text { ind })}{r(\text { ind })}
$$


The values of $\omega$ vary from 1.0 in complete overlapping, to 0.0 in independent occurrence, to -1.0 in complete exclusion.

\section{RESULTS}

\section{(1) Species overlap}

Table 1 shows the values of $\gamma$ and $\omega$ of each two species combination for the "trunk units" and for " $200 \mathrm{~cm}$." The index of the "trunk units" was calculated by treating independently all trunk units which permitted measuring the degree of overlap on each trunk unit. For the index of " $200 \mathrm{~cm}$," every four trunk units $(50 \mathrm{~cm} \times 4$ unit= $200 \mathrm{~cm}$ ) from the ground were pooled for all sample trees (4 trunk units $\times 14$ sample trees $\times$ ca. 0.5 (ratio of the number of trunk unit of the Treatment (see Yoshikawa et al., 1986, in detail) which equaled ca. 28 trunk units per unit size of " $200 \mathrm{~cm}$ ")). This analysis indicates the degree of overlapping in relation to vertical distribution.

(i) Shirahoshizo spp.

Indices $\gamma$ and $\omega$ of the "trunk units" for Shirahoshizo spp. were almost 0 and -1 , respectively, with every other species. Therefore Shirahoshizo spp. is distributed almost completely exclusive from other species on trunk units $(50 \mathrm{~cm}$ long). Comparative values with $M$. alternatus were not so small as with other species. The larger values could be accounted for by the distribution of Shirahoshizo spp. and M. alternatus which partly overlapped and coexisted in thick bark (Yoshikawa, 1987). Moreover, since Shirahoshizo spp. emerged only from the lower part of stem, these two species appear to occupy the same habitat.

The indices of " $200 \mathrm{~cm}$ " for Shirahoshizo spp. were also low values, indicating the

Table 1. Values of $\gamma$ and $\omega$ for each two species combination for the unit size (a) "trunk units" and (b) "200 cm"

(a) Trunk unit

\begin{tabular}{|c|c|c|c|c|c|}
\hline \multirow{2}{*}{$\omega$} & \multicolumn{5}{|c|}{$\gamma$} \\
\hline & $S$. spp. & P. $n$. & M.a. & A.g. & $T . f$. \\
\hline Shirahoshizo spp. & - & 0.0001 & 0.0709 & 0.0324 & 0.0119 \\
\hline P. nitidus & -0.9981 & - & $* *$ & $* *$ & 0.2375 \\
\hline M. alternatus & -0.5963 & $* *$ & - & 0.1600 & 0.4346 \\
\hline A. griseus & -0.7322 & $* *$ & -0.2665 & - & 0.2138 \\
\hline$T$. fulvus & -0.9378 & 0.0576 & 0.1400 & -0.0936 & - \\
\hline
\end{tabular}

(b) $200 \mathrm{~cm}$

\begin{tabular}{lccccc}
\hline \multicolumn{1}{c}{$\omega$} & \multicolumn{5}{c}{$\gamma$} \\
\cline { 2 - 6 } & S. spp. & P.n. & M.a. & A.g. & $T . f$. \\
\hline Shirahoshizo spp. & - & 0.0667 & 0.1560 & 0.3181 & 0.1137 \\
P. nitidus & -0.8559 & - & $* *$ & $* *$ & 0.9125 \\
M. alternatus & -0.6494 & $* *$ & - & 0.8214 & 0.9026 \\
A. griseus & -0.2811 & $* *$ & 0.3267 & - & 0.7085 \\
T. fulvus & -0.7766 & 0.7307 & 0.6531 & -0.0092 & - \\
\hline
\end{tabular}

**: did not coexist. 
exclusive distribution from other species. Indices with $T$. fulvus and with $P$. nitidus indicated almost complete exclusive distribution for both the "trunk units" and "200 cm." Such exclusive distribution both vertically and individually indicate niche segregation along an environmental gradient changing with height. On the other hand, indices of " $200 \mathrm{~cm}$ " with A. griseus were not as small which suggests a more independent distribution from each other. The individually exclusive and vertically independent distributions show that the distribution ranges of Shirahoshizo spp. and A. griseus are somewhat overlapping and they occupy different habitat.

(ii) Taenioglyptes fulvus

Both indices, $\gamma$ and $\omega$, of " $200 \mathrm{~cm}$ " for $T$. fulvus with $P$. nitidus and with $M$. alternatus were about 0.9 and 0.7 , respectively. These indices indicate a high degree of overlapping distribution. However, indices of the "trunk units" for these two species showed independent distributions and indicate niche segregation. Both $P$. nitidus and $M$. alternatus were distributed over the same range as $T$. fulvus, but occupied different habitat in each trunk unit, because of their different body sizes. Namely body length of T. fulvus, $M$. alternatus and $P$. nitidus are $1.3-1.9 \mathrm{~mm}, 18-27 \mathrm{~mm}$ and $6.5-7.5 \mathrm{~mm}$, respectively.

With A. griseus, $\gamma$ was 0.7 and $\omega$ was 0 for the " $200 \mathrm{~cm}$ " unit and 0.2 and -0.1 for the "trunk units." They were distributed independently along the stem and exclusive at each trunk unit. The values of $\gamma$ and $\omega$ were larger than those in the case between Shirahoshizo spp. and A. griseus. Therefore A. griseus had a more exclusive relation with Shirahoshizo spp. than with T. fulvus.

(iii) Acanthocinus griseus and Monochamus alternatus

The vertical distribution ranges of $A$. griseus and $M$. alternatus overlapped each other, as indicated by $\gamma$ and $\omega$ values of 0.8 and 0.3 for the " $200 \mathrm{~cm}$ " units as mentioned previously by Yoshikawa (1987). On the other hand, they were exclusive at each trunk unit, as shown by low values of $\gamma$ and $\omega$. The exclusive relation between these two species was strongest among the combinations between species distributed at the same range along the stem. This suggests that $A$. griseus and $M$. alternatus used the same habitat and competed directly with each other.

\section{(2) Species interaction}

(i) Shirahoshizo spp.

The emergence density associated with $M$. alternatus was higher than the density of Shirahoshizo spp. by itself (Fig. 1). As mentioned above, $M$. alternatus can inhabit the distribution range of Shirahoshizo spp. M. alternatus appears to select trunk units specially suitable for Shirahoshizo spp. at the bottom of stem. However, emergence decreased where $A$. griseus emerged. Since the emergence density of Shirahoshizo spp., where the three species coexisted, was almost the same as the density by itself, A. griseus obviously did not compete directly with Shirahoshizo spp. in the lower part of stem, but occupied the habitat that was not suitable for Shirahoshizo spp. Above $2.5 \mathrm{~m}$ from the ground, emergence was restricted by some environmental conditions changing with height.

Judging from the emergence density of Shirahoshizo spp. below $2.5 \mathrm{~m}$, there were several habitats grouped by their quality for larval development. These were the superior habitat for Shirahoshizo spp. intruded by $M$. alternatus, the unsuitable habitat mainly consumed by $A$. griseus and the medium habitat which could be used by all three species. Since the number of trunk units where the three species could coexist 


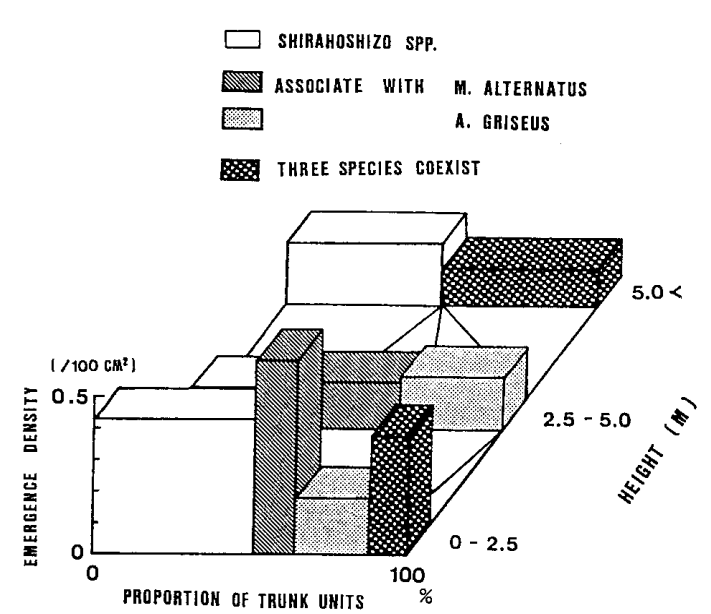

Fig. 1. Vertical changes in the emergence density of Shirahoshizo spp. Percentage on abscissa is the proportion trunk units with recorded emergence corresponding to each species indicated by the pattern of each column.

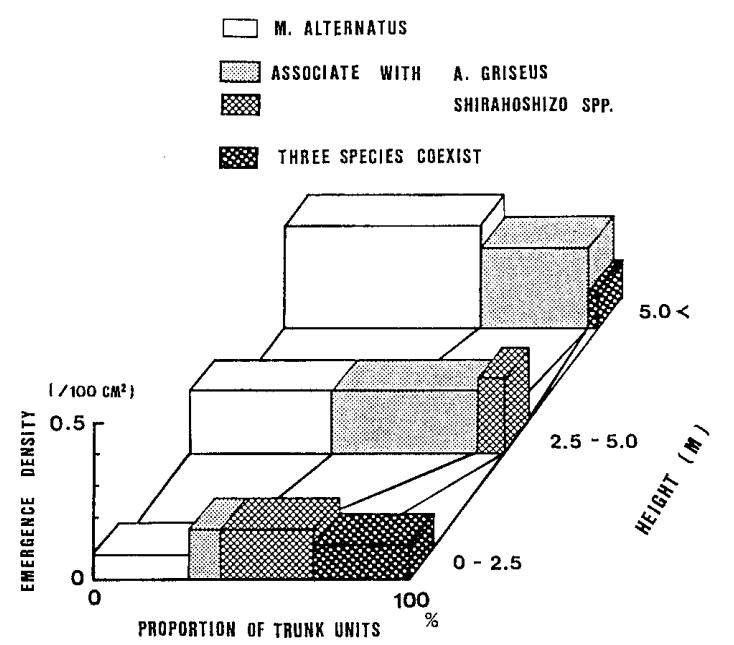

Fig. 2. Vertical changes in the emergence density of $M$. alternalus.

was much smaller than the number of units where only Shirahoshizo spp. emerged, apparently most of these two Cerambycidae are excluded in the medium habitat by this bark weevil.

\section{(ii) Monochamus alternatus}

The number of trunk units used by $M$. alternatus increased with height -10 trunk units at $0-2.5 \mathrm{~m}, 24$ at $2.5-5.0 \mathrm{~m}$ and 32 at more than $5.0 \mathrm{~m}$ in height. Habitats were more preferable for this species on the upper stem. Moreover the emergence density and the proportion of the trunk units monopolized by this species increased with height (Fig. 2). This may be the result of increased attack as habitat improved.

Above $2.5 \mathrm{~m}$ height, the main species associated with $M$. alternatus was $A$. griseus. The emergence density of $M$. alternatus did not change at any height with the presence of $A$. griseus. Therefore $M$. alternatus larvae were not affected by other coexisting species, because of their niche segregation or superiority in interspecific competition.

(iii) Acanthocinus griseus

The proportion of the trunk units with no associate species did not change with height, but the emergence density of $A$. griseus from these trunk units showed a remarkable increase with height (Fig. 3). However, the emergence of $A$. griseus from trunk units containing $M$. alternatus remained almost constant at every height. An extreme difference in emergence density of $A$. griseus was observed between trunk units monopolized by $A$. griseus and those containing $A$. griseus and $M$. alternatus above $5 \mathrm{~m}$ from the ground. This result seemed to suggest that $A$. griseus cannot compete with $M$. alternatus.

Emergence density below $2.5 \mathrm{~m}$ did not change with the presence of associated species. Therefore, A. griseus competed with $M$. alternatus in the upper part of the stem, and was independent of other species in the lower part.

Although the quality of the habitat for $A$. griseus apparently was abruptly improved 


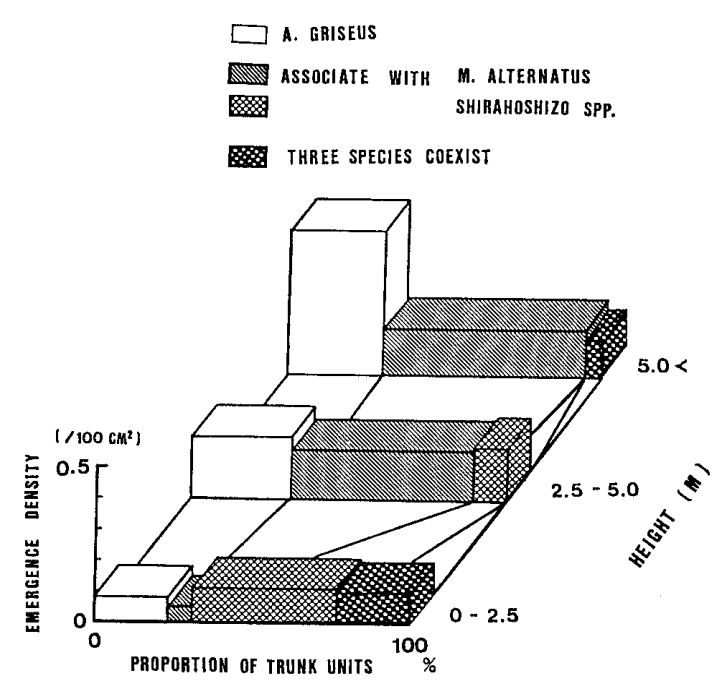

Fig. 3. Vertical changes in the emergence density of $A$. griseus.

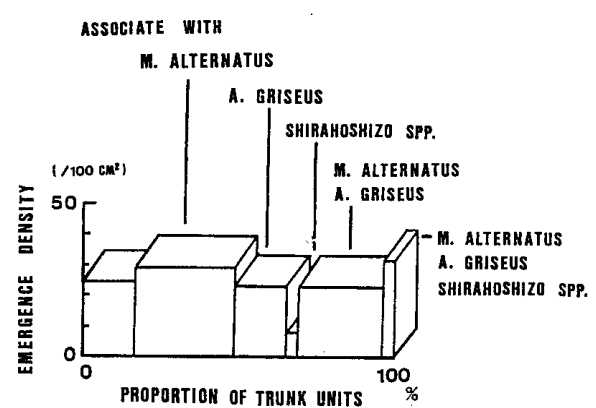

Fig. 4. The emergence density of $T$. fulvus in relation to associate species.

with height judged by emergence density, the number of trunk units used by $A$. griseus did not change with height, namely 13, 19 and 17 trunk units at each height level from the ground. Therefore the oviposition and larval development of this species was controlled by factor(s) independent of the height and the quality of the habitat.

(iv) Taenioglyptes fulvus

Most of the trunk units above $2.0 \mathrm{~m}$ from the ground were consumed by this species (Yoshikawa, 1987). But only a small proportion of the trunk units was monopolized by $T$. fulvus (Fig. 4). The emergence density of this species decreased where Shirahoshizo spp. emerged, but was not affected by coexistence with Cerambycidae. Therefore, I concluded that the part of the stem used by Shirahoshizo spp. was not suitable habitat for $T$. fulvus and moreover, as these two species were distributed exclusive (Table 1), severe competition between them decreased the emergence density of this species in such unsuitable habitat. However, in the habitat available for their development, they seldom suffered from competition of other species. The most acceptable explanation of this phenomenon is the difference of their niche caused by their body sizes.

The mean emergence densities of $T$. fulvus were 59.68 and 49.08 individuals per $100 \mathrm{~cm}^{2}$ where $P$. nitidus absent and present, respectively. Densities of $P$. nitidus was 0.39 individuals per $100 \mathrm{~cm}^{2}$ whether $T$. fulvus was absent or present. This relation coincided with the results of species overlap mentioned above, namely that these two species are distributed independently from each other in spite of having the same distribution along the stem.

\section{DISCUSSION}

The insects that work in the cambium fall naturally into two groups: those that spend all, and those that spend only a part of their developmental period in this region (Graham, 1925). The first group is typified by the bark beetles and weevils ( $T$. fulvus, Shirahoshizo spp. and $P$. nitidus). These insects are dependent throughout their life 
upon the fresh green tissues of the inner bark and cambium. This requirement limits specifically the length of the feeding period and the distribution range of these insects. They must of necessity develop rapidly while the perishable material upon which they feed remains suitable for them. They must make use of it before it becomes dry, and before other organisms have altered its chemical and physical nature (RuDinsky, 1962). The second group of cambium feeders is somewhat less limited in its food requirements. This group is typified by many Cerambycidae and Buprestidae ( $M$. alternatus and $A$. griseus). These insects require green tissue only for their young stages, but are able, after getting well started, to continue their growth and development in the solid wood. Apparently their digestive powers increase with age until finally they are able to digest cellulose and convert it into living protoplasm. Such differences must affect their oviposition behavior and the ability of interspecific competition in the larval stage.

Among the subcortical species emerged from pine bait logs in this experiment, Shirahoshizo spp. and T. fulvus could attack pine trees throughout the growing season (Yoshikawa et al., 1986). Then damaged or dead pine trees in this study area could not be delivered from attacks by these two species. Therefore, the basic distribution pattern of the subcortical community in Pinus densiflora was T. fulvus in the upper part of stem with smooth bark and Shirahoshizo spp. at the bottom part of stem with rough bark. Both species fed in the phloem layer all their developmental period and, there was a complete niche segregation between them. The schematic distributions of each subcortical species are shown in Fig. 5.

Pine trees that lost their vigor in the spring were attacked by $P$. nitidus, T. fulvus and Shirahoshizo spp. The habitat segregation between Shirahoshizo spp. and Pissodes spp. in the pine trunk was reasonably explained by bark thickness (KOBAYASHI, 1975). Judging from their interaction as mentioned above, $T$. fulvus and $P$. nitidus occurred on the upper part with thin bark, but they had different niche requirements and, as a result, were distributed independently with each other in each trunk unit. The range of emergence of $T$. fulvus was slightly higher than that of $P$. nitidus.

When pine trees were weakened in the summer, two Cerambycidae participated in the subcortical community instead of $P$. nitidus. $M$. alternatus and $A$. griseus fed on

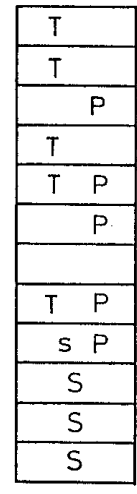

spring

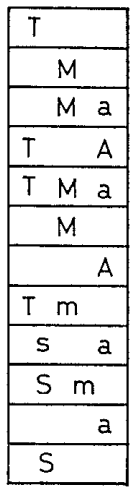

summer

Fig. 5. Imaginary distribution of the subcortical insects in the trunk of $P$. densiflora. Spring and summer are the periods of insect attack. T: T. fulvus, P: P. nitidus, $\mathrm{S}$ and s: Shirahoshizo spp., M and m: M. alternatus, A and a: A. griseus. Capitals and small letters are the high and low emergence density, respectively. 
both phloem and sapwood and showed an overlapping distribution. Consequently, they competed severely, and usually $M$. alternatus won. KовAYAshi (1975) reported that Arhopalus rusticus L. occurred in the middle part of trunk after being inhibited from the lower part by aerial spray of insecticide to protect from the attack of $M$. alternatus (NAKAhara et al., unpublished). Therefore, $M$. alternatus was the strongest competitor among the three pine sawyers, $M$. alternatus, $A$. griseus and $A$. fusticus.

The competitive exclusion principle states that two species cannot coexist on the same limiting resource. Then when two species compete, natural selection occurs in both of them, with optimal evolutionary strategy oriented to avoid interspecific competition. One strategy is character divergence. Yoshida and TsudA (1978) suggested that the difference in body length of three dominant pine sawyers, $M$. alternatus, $A$. griseus and Arhopalus rusticus which were $18-27 \mathrm{~mm}, 9-13 \mathrm{~mm}$ and $10-27 \mathrm{~mm}$, respectively, was evidence of character divergence, that is, reflection of different life style and different niche occupation. For example, $M$. alternatus clearly showed a suitable height in their vertical distribution (Yoshikawa, 1987), but A. griseus did not show such vertical distribution. It has a wide range of distribution being independent from the environmental conditions associated with height. Moreover, on the lower part of the stem, A. griseus was able to use part of the trunk not suitable for Shirahoshizo spp., thus avoiding direct competition.

The basic pattern of distribution for the subcortical insects was exclusion with respect to each other, because most of their food was the same quality, they could utilize almost the same habitat under bark. However, when body size differed greatly, they could coexist, for example $T$. fulvus and other species. Because large sized species fed in the cambium but left fairly large amount of unutilized food, small species were able to develop in it.

\section{ACKNOWLEDGEMENTS}

I wish to express my sincere thanks to Dr. G. D. Amman, Project Leader of USDA, for his invaluable advice and critical reading of this manuscript. Thanks are also due to Dr. H. TAkEdA, Forest Ecology Laboratory of Kyoto University, Dr. K. Soné, Forestry and Forest Products Research Institute, and Mr. E. Shibata, Nara Forest Experiment Station, for their encouragement and helpful criticism in all aspects of this study.

\section{REFERENCES}

BeAver, R. A. (1966) The biology and immature stages of two species of Medetera (Diptera: Dolichopodidae) associated with the bark beetle Scolytus scolytus (F.). Proc. R. Entomol. Soc. Lond. A 41 : 145-154.

Berryman, A. A. (1966) Studies on the behavior and development of Enoclerus lecontei (WolcotT), a predator of the Western pine beetle. Can. Ent. 98: 519-526.

Berryman, A. A. (1967) Estimation of Dendroctonus brevicomis (Coleoptera: Scolytidae) mortality caused by insect predators. Can. Ent. 99: 1009-1014.

Berryman, A. A. (1968) Distribution of Scolytus ventralis attacks, emergence, and parasites in grand fir. Can. Ent. 100 : 57-69.

Berryman, A. A. (1973) Population dynamics of the fir engraver, Scolytus ventralis (Coleoptera: Scolytidae) I. Analysis of population behavior and survival from 1964 to 1971. Can. Ent. 105: 1465-1488.

Cole, L. M. (1949) The measurement of interspecific association. Ecology 30: 411-424.

Dice, L. R. (1945) Measures of the amount of ecological association between species. Ecology 26: 297302. 
Graham, S. A. (1925) The felled tree trunks as an ecological unit. Ecology 6: 397-411.

IwAo, S. (1977) Analysis of spatial association between two species based on interspecies mean crowding. Res. Popul. Ecol. 18: 243-260.

Kiyohara, T. and Y. Tokushige (1971) Inoculation experiments of nematode, Bursaphelenchus sp., onto pine trees. J. Jpn. For. Soc. 51: 193-195.

Koвayashi, F. (1975) Studies on the methods for estimating density and distribution of forest insect populations (I). Distribution of coleopterous borers in pine. Bull. Gov. For. Stn. 274: 85-124 (in Japanese with an English summary).

Koвayashi, F. (1978) Pine bark beetle problem in Japan, referring to the discovery of the pine wood nematode, Bursaphelenchus lignicolus (Mamrya \& Kryohara). Anz. Scädlingskde., Pflanzenschutz, Umweltschutz 51 : 76-79.

Kobayashi, F. (1981) Review on the pine mortality and its research in Japan. Proc. XVIII IUFRO World Congr. Div. 2: 261-263.

Levins, R. (1968) Evolution in Changing Environments. Princeton Univ. Press, Princeton, 120 pp.

Mamrya, Y. and N. Enda (1972) Transmission of Bursaphelenchus lignicolus (Nematoda: Aphelenchoidea) by Monochamus alternatus (Coleoptera: Cerambycidae). Nematologica 18: 159-162.

MamiYa, Y. and T. KiYohara (1972) Description of Bursaphelenchus lignicolus n. sp. (Nematoda: Aphelenchoididae) from pine wood and histophathology of nematode-infested trees. Nematologica 18: 120-124.

MorishitA, M. (1959) Measuring of interspecific association and similarity between communities. Mem. Fac. Sci. Kyushu Univ., Ser. E (Biol.) 3: 65-80.

Rudinsky, J. A. (1962) Ecology of Scolytidae. Ann. Rev. Entomol. 7: 327-348.

Yoshida, T. and Y. Tsuda (1978) The emergence of insects from dying pine trees. Bull. Fac. Agric. Univ. Okayama 51: 17-21 (in Japanese with an English summary).

Yoshikawa, K. (1977) Population study of pine bark weevils (Coleoptera: Curculionidae) in bait logs. Appl. Ent. Zool. 12: 9-17.

Yoshikawa, K. (1987) A study of the subcortical insect community in pine trees. II. Vertical distribution. Appl. Ent. Zool. 22: 195-206.

Yoshimawa, K., H. Takeda, S. Soné and E. Shibata (1986) A study of the subcortical insect community in pine trees. I. Oviposition and emergence periods of each species. Appl. Ent. Zool. 21: 258-268. 